\title{
No Finite Time Blowup for 3D Incompressible Navier Stokes Equations via Scaling Invariance
}

\author{
Terry E. Moschandreou \\ Brooklyn College, Toronto Ontario, Canada \\ ${ }^{*}$ Corresponding Author: tv42022@tvdsb.ca
}

Received March 4, 2021; Revised May 5, 2021; Accepted May 16, 2021

Cite This Paper in the following Citation Styles

(a): [1] Terry E. Moschandreou, "No Finite Time Blowup for 3D Incompressible Navier Stokes Equations via Scaling Invariance," Mathematics and Statistics, Vol.9, No.3, pp. 386-393, 2021. DOI: 10.13189/ms.2021.090321

(b): Terry E. Moschandreou, (2021). No Finite Time Blowup for 3D Incompressible Navier Stokes Equations via Scaling Invariance. Mathematics and Statistics, 9(3), 386-393. DOI: 10.13189/ms.2021.090321

Copyright $\odot 2021$ by authors, all rights reserved. Authors agree that this article remains permanently open access under the terms of the Creative Commons Attribution License 4.0 International License

\begin{abstract}
The problem to The Clay Math Institute "NavierStokes, breakdown of smooth solutions here on an arbitrary cube subset of three dimensional space with periodic boundary conditions is examined. The incompressible Navier-Stokes Equations are presented in a new and conventionally different way here, by naturally reducing them to an operator form which is then further analyzed. It is shown that a reduction to a general 2D N-S system decoupled from a 1D non-linear partial differential equation is possible to obtain. This is executed using integration over $\mathrm{n}$-dimensional compact intervals which allows decoupling. The operator form is considered in a physical geometric vorticity case, and a more general case. In the general case, the solution is revealed to have smooth solutions which exhibit finite-time blowup on a fine measure zero set and using the Prékopa-Leindler and Gagliardo-Nirenberg inequalities it is shown that for any non zero measure set in the form of cube subset of 3D there is no finite time blowup for the starred velocity for large dimension of cube and small $\delta$. In particular vortices are shown to exist and it is shown that zero is in the attractor of the 3D Navier-Stokes equations.
\end{abstract}

Keywords Incompressible, Navier-Stokes, Blowup, Gagliardo-Nirenberg, Millenium Problem

\section{Introduction}

The question of whether the solutions to the 3-D- Incompressible N-S equations are globally regular or demonstrate finite time blowup has been a long going debate in mathematics and in general the scientific communities. The Millennium problem posed by the Clay Institute [1] is asking for a proof of one of the above conjectures. Seminal papers conducted by Jean Leray [2, 3, 4] proved that there exists a global (in time) weak solution and a local strong solution of the initial value problem when the domain is all of $\mathbb{R}^{3}$, that is solutions up to some finite $T^{*}$ on an interval $\left[0, T^{*}\right]$. While specific cases have approached answers in unique cases, the question of whether there is a unique solution for all instants of time, (ie. a global unique solution) is presently open. It has been shown that there exists a unique global solution for the $2 \mathrm{D}$ plane-parallel N-S equations [5, 6]. While at first glance, the NSEs appear as a compact set of PDEs, the fascination with these Partial Differential Equations is only increased by the fact that the nonlinearity of the ensuing expanded equations, appear to be connected with notions of highly chaotic turbulence and vorticity[7, 8, 9]. Since the announcement of the Millennium Problem, several results have attempted to comment on the existence and uniqueness of the NSEs. One particularly recent result by Kyritsis noted that there existed indications towards establishing a regularity of solutions regarding the Euler Equations and NSEs more generally; this utilized the conservation of particles[10]. In the present work, to the best of the authors' knowledge, the procedure revealed here has not been previously observed in the literature on the question of Incompressible N-S 3-D existence of unique global solutions, except for compressible flows in [11, 12, 13]. First, a cube in $\mathbb{R}^{3}$ with boundary conditions that generate a vortex is considered , and an attempt has been made to naturally reduce the 3-D incompressible NSEs to a one component decoupled velocity field solution under scale invariant transformations, with a separate 2-component velocity field solution. For the variable $z$ component, in particular, a form of solution is extracted in the 
analysis presented using the divergence form of Green's identity, Ostogradsky's theorem. The decomposition method using Geometric Algebra is used together with a main result that a bound for $\left\|u_{z}\right\|_{\infty}$ implies bounds for all derivatives. [14] [18]

\section{Model}

The 3D incompressible unsteady Navier-Stokes Equations (NSEs) in Cartesian coordinates may be listed below in compactified form for the velocity field $\mathbf{u}^{*}=u^{* i} \overrightarrow{\mathbf{e}}_{i}, u^{* i}=$ $\left\{u_{x}^{*}, u_{y}^{*}, u_{z}^{*}\right\}$ :

$$
\rho\left(\frac{\partial}{\partial t^{*}}+u^{* j} \nabla_{* j}\right) u_{i}^{*}-\mu \nabla_{*}^{2} u_{i}^{*}+\nabla_{* i} P^{*}=\rho F_{i}^{*}
$$

where $\rho$ is constant density, $\mu$ is dynamic viscosity, $\mathbf{F}^{*}=F^{* i} \overrightarrow{\mathbf{e}}_{i}$ are the body forces on the fluid. In some cases, it may be elected to reparametrize the components of the velocity vector, and pressure to $\mathbf{u}=(u)^{i} \overrightarrow{\mathbf{e}}_{i}, \mathbf{P}=(P)^{i} \overrightarrow{\mathbf{e}}_{i}$, coordinates $\mathbf{x}_{i}$ and time $t$ according to the following form utilizing the non-dimensional quantity $\delta$ (assumed negative):

$$
u_{i}^{*}=\frac{1}{\delta} u_{i}, P_{i}^{*}=\frac{1}{\delta^{2}} P_{i}, x_{i}^{*}=\delta x_{i}, t^{*}=\delta^{2} t
$$

The continuity equation in Cartesian co-ordinates, is

$$
\nabla^{i} u_{i}=0
$$

\subsection{Data}

Eq.(1), together with Eq.(3) and using the initial condition of $\overrightarrow{u^{*}}\left(\overrightarrow{x^{*}}, 0\right)=\vec{\xi}\left(\overrightarrow{x^{*}}\right)$ such that $\nabla \cdot \vec{\xi}=0$ encompass the NSEs along with an incompressible initial condition. Ensuring periodic boundary conditions specified in [1] defined on a cube domain $\Omega$ in $\mathbb{R}^{3}$ is referred to as the periodic BVP for the NSEs in $\mathbb{R}^{3}$. See Fig. 1 below for lattice geometry of flow problem. I take the cube to be centred at the origin.

\section{Application}

Using Eq2 above, multiplying the first two components of scale invariant Eq 1 by Cartesian unit vectors $\vec{i}=(1,0,0)$, $\vec{j}=(0,1,0)$ respectively and adding modified equations within the set Eq1 give the following equations, for the resulting composite vector $\vec{b}=\frac{1}{\delta} u_{x} \vec{i}+\frac{1}{\delta} u_{y} \vec{j}$,

$$
\begin{gathered}
\frac{1}{\delta^{2}} \frac{\partial \vec{b}}{\partial t}+\frac{u_{x}}{\delta^{2}} \frac{\partial \vec{b}}{\partial x}+\frac{u_{y}}{\delta^{2}} \frac{\partial \vec{b}}{\partial y}-\frac{\mu}{\rho \delta^{2}} \nabla^{2} \vec{b} \\
+\frac{1}{\delta^{3} \rho} \frac{\partial P}{\partial x} \vec{i}+\frac{1}{\delta^{3} \rho} \frac{\partial P}{\partial y} \vec{j}=\vec{F}_{T}
\end{gathered}
$$

where $\vec{F}_{T}=\frac{1}{\delta^{2}}$. Multiplying Eq. 4 by $\delta^{3}$ and by $u_{z}^{*}$ and, the $z^{*}$ component of Eq 1 by $\delta^{3}$ and by $\vec{b}$, (again using Eq,2), the addition of the resulting equations [11, 12, 13] recalling the product rule, produces a form as displayed below in Eq 5 , where $\vec{a}=u_{z} \vec{b}$. The nonlinear inertial term when added to $\vec{b} \nabla u_{z} \cdot \vec{b}$ and factoring out $\vec{b}$ gives, $\vec{b} \cdot \nabla \vec{a}$. Here $\nabla u_{z} \vec{b}$ is a dyadic.

$$
\begin{gathered}
\frac{\partial \vec{a}}{\partial t}+\vec{b} \cdot \nabla \vec{a}-\frac{\mu}{\rho} u_{z} \nabla^{2} \vec{b}+\frac{1}{\delta} \frac{1}{\rho} u_{z} \nabla_{x y} P \\
+\vec{b} \frac{1}{\rho} \frac{\partial P}{\partial z}+\delta^{2} u_{z} \vec{F}_{T}+\delta^{3} F_{z} \vec{b}-\frac{\mu}{\rho} \vec{b} \nabla^{2} u_{z}=0
\end{gathered}
$$

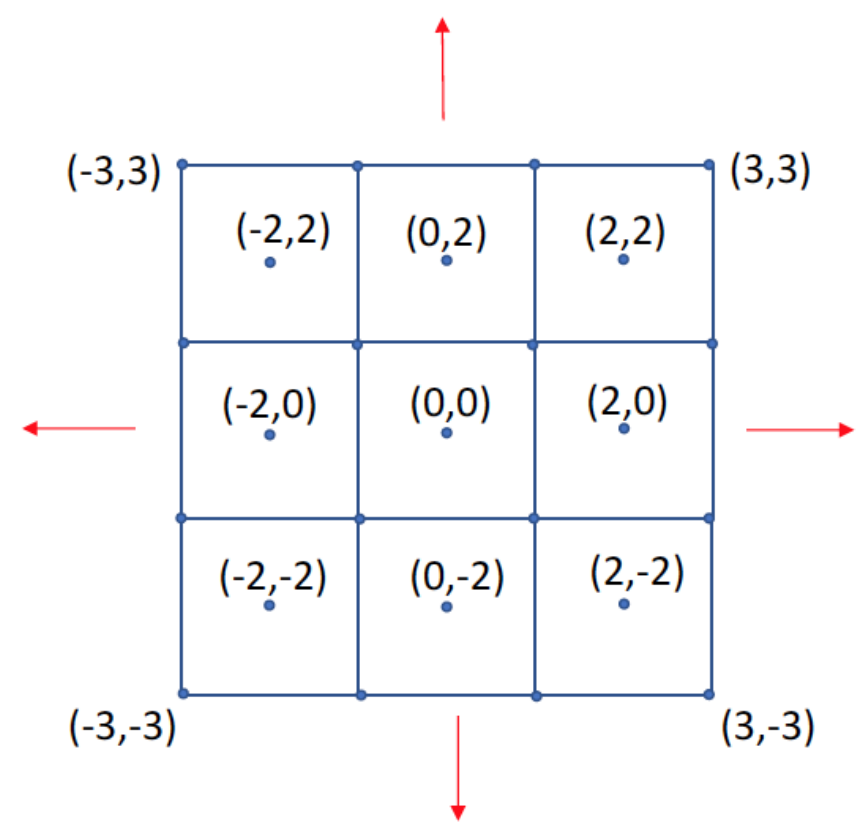

Figure 1. Vortex generation in a 2-D Lattice.

Multiplying Eq 5 by $u_{z}$ and adding to it $u_{z} \vec{b} \nabla \cdot \vec{a}$ gives:

$$
\begin{gathered}
u_{z} \frac{\partial \vec{a}}{\partial t}+\vec{a} \cdot \nabla \vec{a}-\frac{\mu}{\rho} u_{z}^{2} \nabla^{2} \vec{b}+\frac{1}{\delta \rho} u_{z}^{2} \nabla_{x y} P+ \\
\vec{b} \frac{1}{\rho} u_{z} \frac{\partial P}{\partial z}+\delta^{2} u_{z}^{2} \vec{F}_{T}+\delta^{3} u_{z} F_{z} \vec{b}+ \\
u_{z} \vec{b} \nabla \cdot \vec{a}-\frac{\mu}{\rho} u_{z} \vec{b} \nabla^{2} u_{z}=0
\end{gathered}
$$

At this point the $z$ component of the external force, $F_{z}$, is assumed to be offset exactly by $F_{T_{1}}, F_{T_{2}}$. In this paper Eq6 6 is solved, instead of $\mathrm{Eq} 5$ For the $\varepsilon$-periodic solution, it is proposed that integration of divergence or curl of Eq 6 over an arbitrary small volume is equivalent to integration of divergence or curl of $\mathrm{Eq} 5$ for the same volume. That is the extra term's divergence or curl in Eq 6 when integrated is negligible on set of measure zero. A necessary condition that the form of Eq. 5. call it $L_{1}=0$, is that both the divergence and curl of $L_{1}$ be zero and upon integrating over a volume $U$, we have the function of $t$ which we call $C_{S}(t)$ which consists of the force term $F_{T}$. The same is true for the non $\varepsilon$ - periodic case where the justification of using Eq 6 instead of 5 will be based on the periodicity of the flow on an interval in $\mathbb{R}^{3}$. It can be seen that for the general non zero measure set, upon separately taking the divergence and curl of Eq. 6 and integrating over the volume $U$ and using first Ostogradsky's theorem and the fact that the velocities are periodic on the interval in $\mathbb{R}^{3}$, we have that the extra term $u_{z} \vec{b} \nabla \cdot \vec{a}=\vec{a} \nabla \cdot \vec{a}$ has an integral of it's divergence equal to zero. Proof is straightforward upon taking the divergence and integrating. Next for the curl of the same term and integrating over the same volume we use the fact that $\int_{U} \nabla \times \vec{F} d V=\iint_{\partial U} \vec{n} \times \vec{F} d S$. This contribution is also zero due to periodicity of velocities on interval. 
Theorem 1. Reduced structure form of 3-D Navier-Stokes Equations

The 3-D Incompressible Navier Stokes equations can be reduced to a simple form as,

$$
\mathscr{G}=\frac{\vec{f}}{\vec{f} \cdot \vec{b}} \cdot Q
$$

where $\mathscr{G}$ is the nonlinear partial differential operator given by $E q .12, Q$ is the two dimensional Navier-Stokes operator acting on the vector $\vec{b}$ defined in the brackets of $E q \cdot 17$ and $\vec{f}=\vec{a} \cdot \nabla \vec{a}$. The terms $\Omega_{i}$ contained in $\mathscr{G}$ are defined after $E q[10$ and are part of the proof of present Theorem 1 .

Proof. Taking the geometric product with the inertial vector term in the previous equation Eq 6 given by $\vec{f}=\vec{a} \cdot \nabla \vec{a}$, it can be shown that in the context of Geometric Algebra [11, 13], the following scalar and vector grade equations arise:

$$
\begin{gathered}
\text { SCALAR } \\
\vec{f} \cdot\left(u_{z}^{2} \frac{\partial \vec{b}}{\partial t}+u_{z} \vec{b} \frac{\partial u_{z}}{\partial t}\right)+\|\vec{f}\|^{2}+u_{z}(\vec{f} \cdot \vec{b}) \nabla \cdot \vec{a}= \\
\frac{\mu}{\rho} u_{z}^{2} \vec{f} \cdot \nabla^{2} \vec{b}-\frac{1}{\delta \rho} u_{z}^{2} \vec{f} \cdot \nabla_{x y} P- \\
\vec{f} \cdot \vec{b} \frac{1}{\rho} u_{z} \frac{\partial P}{\partial z}+\frac{\mu}{\rho} u_{z} \nabla^{2} u_{z}(\vec{f} \cdot \vec{b})+\vec{f} \cdot(\text { Force Terms) }
\end{gathered}
$$

$$
\begin{gathered}
\text { VECTOR } \\
u_{z}^{2} \frac{\partial \vec{b}}{\partial t}+\vec{a} \frac{\partial u_{z}}{\partial t}+u_{z} \vec{b} \nabla \cdot \vec{a}=\frac{\mu}{\rho} u_{z}^{2} \nabla^{2} \vec{b}- \\
\frac{1}{\delta \rho} u_{z}^{2} \nabla_{x y} P-\vec{b} \frac{1}{\rho} u_{z} \frac{\partial P}{\partial z}+ \\
\frac{\mu}{\rho} u_{z} \vec{b} \nabla^{2} u_{z}+\text { Force Terms }
\end{gathered}
$$

Taking the divergence of the vector equation $\mathrm{Eq} 8$, recalling the product rule, and defining the new term $H=\left(u_{z} \vec{b}\right.$. $\vec{f}) /\left(\partial_{t} u_{z}\right)$ (After taking divergence multiply new equation by $H)$, results in an expression which may be combined with the usage of the scalar equation $\mathrm{Eq} 7$ to produce:

$$
\begin{aligned}
& H \nabla u_{z}^{2} \cdot \frac{\partial \vec{b}}{\partial t}+u_{z}^{2} H \frac{\partial}{\partial t}(\nabla \cdot \vec{b})+ \\
& H \vec{a} \cdot \nabla\left(\frac{\partial u_{z}}{\partial t}\right)+H \nabla \cdot\left(\vec{b} u_{z} \nabla \cdot \vec{a}\right)-\frac{\mu}{\rho} H \nabla \cdot\left(u_{z}^{2} \nabla^{2} \vec{b}\right)+ \\
& H \nabla \cdot\left(\frac{1}{\delta \rho} u_{z}^{2} \nabla_{x y} P+\vec{b} \frac{1}{\rho} u_{z} \frac{\partial P}{\partial z}\right)- \\
& H \frac{\mu}{\rho} u_{z} \nabla^{2} u_{z} \nabla \cdot \vec{b}-H \frac{\mu}{\rho} \nabla\left(u_{z} \nabla^{2} u_{z}\right) \cdot \vec{b}+H \text { Div of Force Terms = } \\
& \vec{f} \cdot\left(u_{z}^{2} \frac{\partial \vec{b}}{\partial t}+u_{z} \vec{b} \frac{\partial u_{z}}{\partial t}-\frac{\mu}{\rho} u_{z} \vec{b} \nabla^{2} u_{z}\right)+ \\
& \|\vec{f}\|^{2}-\frac{\mu}{\rho} u_{z}^{2} \vec{f} \cdot \nabla^{2} \vec{b}+\frac{1}{\delta \rho} u_{z}^{2} \vec{f} \cdot \nabla_{x y} P+\frac{1}{\rho} \vec{f} \cdot \vec{b} u_{z} \frac{\partial P}{\partial z}+\vec{f} \cdot \text { Force Terms }
\end{aligned}
$$

Continuing with the previous paragraph we use the common term $u_{z}(\vec{f} \cdot \vec{b}) \nabla \cdot \vec{a}$ appearing in Eq 7 and in the new equation where we took divergence of $\mathrm{Eq} 8$ and multiplied by $H$. This term in $\mathrm{Eq} 8$ is $\vec{a} \frac{\partial u_{z}}{\partial t}$. Upon a division of the preceding equation
9 by $u_{z}^{2} H$, it can be seen to result in the general form:

$$
\begin{aligned}
& \Omega_{5}+\frac{\partial Y}{\partial t}-G+\Omega_{1}- \\
& \frac{\vec{f}}{\vec{b} \cdot \vec{f}} \cdot\left(F \vec{f}+\frac{1}{\delta \rho} \frac{\partial u_{z}}{\partial t} u_{z}^{-1} \nabla_{x y} P+\frac{\partial u_{z}}{\partial t} u_{z}^{-1} \frac{\partial \vec{b}}{\partial t}-\right. \\
& \left.\frac{\mu}{\rho} \frac{\partial u_{z}}{\partial t} u_{z}^{-1} \nabla^{2} \vec{b}\right)-\Omega_{3}+\Omega_{4}+ \\
& \Omega_{6}+\Omega_{7}-\Omega_{8}+u_{z}^{-2} \nabla \cdot\left(\frac{1}{\delta \rho} u_{z}^{2} \nabla_{x y} P+\vec{b} \frac{1}{\rho} u_{z} \frac{\partial P}{\partial z}\right)- \\
& u_{z}^{-2} \frac{\mu}{\rho} \nabla \cdot\left(u_{z} \vec{b} \nabla^{2} u_{z}\right)+\text { Force terms }=0
\end{aligned}
$$

where for brevity, the following symbols have been defined:

$$
\begin{gathered}
\Omega_{1}=u_{z}^{-2} \vec{a} \cdot \nabla\left(\frac{\partial u_{z}}{\partial t}\right), \Omega_{3}=u_{z}^{-2} \frac{\mu}{\rho} \nabla \cdot\left(u_{z}^{2} \nabla^{2} \vec{b}\right), \\
\Omega_{4}=u_{z}^{-2} \nabla \cdot\left(\vec{b} u_{z} \nabla \cdot \vec{a}\right), \Omega_{5}=u_{z}^{-2} \nabla u_{z}^{2} \cdot \frac{\partial \vec{b}}{\partial t}, Y=\nabla \cdot \vec{b} \\
F=u_{z}^{-3} \frac{\partial u_{z}}{\partial t}, G=\frac{1}{u_{z}^{2}}\left(\frac{\partial u_{z}}{\partial t}\right)^{2}, \\
\Omega_{6}=\frac{\mu}{\rho} \frac{\partial u_{z}}{\partial t} u_{z}^{-2} \nabla^{2} u_{z}, \Omega_{7}=u_{z}^{-2} \nabla \cdot\left(\vec{b} \frac{1}{\rho} u_{z} \frac{\partial P}{\partial z}\right), \\
\Omega_{8}=\frac{1}{\rho} u_{z}^{-2} \frac{\partial u_{z}}{\partial t} \frac{\partial P}{\partial z}
\end{gathered}
$$

The divergence of $\vec{F}_{T}$ is assumed to be zero. It can be seen that the expression beginning with parentheses may be abbreviated into a nonlinear vector operator $\mathbf{Q}$ and so $\mathrm{Eq} 10$ can be written compactly as:

$$
\mathscr{G}\left(u_{z}, \vec{b}\right)=\frac{\vec{f} \cdot \mathbf{Q}\left(u_{z}, \frac{\partial u_{z}}{\partial t} \vec{b}, \vec{b}, \frac{\partial \vec{b}}{\partial t}, \nabla_{x y} P, \vec{F}_{T}\right)}{\vec{f} \cdot \vec{b}}
$$

where $\mathscr{G}$ is the non-linear operator associated with remaining part of Eq 10 . Utilizing the continuity $\mathrm{Eq} 3$, it can be seen that the operator $\mathscr{G}$ is given by the following expression:

$$
\begin{gathered}
\mathscr{G}\left(u_{z}, \vec{b}\right)=\frac{\partial}{\partial t}\left(-\frac{\partial u_{z}}{\partial z}\right)+\Omega_{1}-\Omega_{3}+\Omega_{4}+\Omega_{5}+\Omega_{6}+\Omega_{7}- \\
\Omega_{8}-G+\frac{1}{u_{z}^{2}} \nabla \cdot\left(\frac{1}{\rho} u_{z}^{2} \nabla_{x y} P+\vec{b} \frac{1}{\rho} u_{z} \frac{\partial P}{\partial z}\right)=\frac{\|\mathbf{Q}\|}{\|\vec{b}\|}
\end{gathered}
$$

The nonlinear operator form of the NSEs presented is:

$$
\mathscr{G}=\frac{\vec{f}}{\vec{f} \cdot \vec{b}} \cdot \mathbf{Q}
$$

This completes the proof.

It is observed that Eq. 13 can be expressed as,

$$
\mathscr{G}=\frac{\vec{f}}{\vec{f} \cdot \vec{b}} \cdot \mathbf{Q}=\frac{\|\mathbf{Q}\|}{\|\vec{b}\|}
$$

An important observation is that in $\mathrm{Eq} 12$.

$$
\begin{gathered}
\Omega_{1}+\Omega_{6}-\Omega_{8}-G=\left(\frac{1}{\delta}-1\right) \frac{1}{u_{z}^{2}}\left(\frac{\partial u_{z}}{\partial t}\right)^{2}+ \\
\frac{\mu}{\rho} \frac{1}{u_{z}^{2}}\left(1-\frac{1}{\delta}\right) \frac{\partial u_{z}}{\partial t} \nabla^{2} u_{z}+\frac{1}{\rho u_{z}^{2}}\left(\frac{1}{\delta}-1\right) \frac{\partial u_{z}}{\partial t} \frac{\partial P}{\partial z}
\end{gathered}
$$

Equation 13 displays a general form which may be expanded and analyzed by allowing a geometric assumption to be undergone, or the general case may also be considered. 


\section{Two Cases}

\subsection{The Geometric Case}

As a special case, one may consider the case where:

$$
\vec{f} \cdot \mathbf{Q}=0
$$

This condition means that the Lie Product of the velocity inertia is entirely perpendicular to the Force terms, and thus refers to a vortex fluid scenario. This condition automatically implies $\mathscr{G}=0$ and so,

$$
\begin{gathered}
\mathscr{G}\left(u_{z}, \vec{b}\right)=\frac{\partial}{\partial t}\left(\frac{\partial u_{z}}{\partial z}\right)+\Omega_{1}-\Omega_{3}+\Omega_{4}+\Omega_{5}+\Omega_{6}+\Omega_{7}-\Omega_{8}- \\
G+\frac{1}{u_{z}^{2}} \nabla \cdot\left(\frac{1}{\delta \rho} u_{z}^{2} \nabla_{x y} P+\vec{b} \frac{1}{\rho} u_{z} \frac{\partial P}{\partial z}\right)=0
\end{gathered}
$$

\subsection{The General Case}

For $\mathbf{Q}$, the expressions with $u_{z}$ and it's derivative with respect to $t$ factor out with the exception of tensor product of velocity inertial term in $\vec{b}$ and $\nabla u_{z}$. Thus $\vec{Q}$ is:

$$
\begin{gathered}
\frac{u_{z} \frac{\partial u_{z}}{\partial t}}{u_{z}^{2}} \times \\
{\left[\frac{\partial \vec{b}}{\partial t}+\vec{b} \cdot \nabla \vec{b}-\frac{\mu}{\rho} \nabla^{2} \vec{b}+\frac{1}{\delta \rho} \nabla_{x y} P-\delta^{2} \vec{F}_{T}+\frac{1}{u_{z}} \vec{b} \cdot\left(\vec{b} \otimes \nabla u_{z}\right)\right]=} \\
\frac{1}{u_{z}^{2}} \frac{\partial u_{z}}{\partial t} \vec{b} \cdot\left(\vec{b} \otimes \nabla u_{z}\right)
\end{gathered}
$$

The expression in brackets in $\mathrm{Eq} 17$, ( for $z^{*} \in \mathbb{R}$, consists of the 2-D "plane-parallel" Navier-Stokes Equations and it is well known that if all data of problem are independent of one of $x, y, z$,then the BVP in Eqs.1. Eqs 3, has a unique solution for all instants of time with no restrictions on smallness of $\vec{F}_{T}, \vec{\xi}$ or the domain $\Omega$.[6]. As a result $\mathbf{Q}=\frac{1}{u_{z}^{2}} \frac{\partial u_{z}}{\partial t} \vec{b} \cdot\left(\vec{b} \otimes \nabla u_{z}\right)$.

Using Green's identity, for divergence, also known as Ostogradsky's Theorem, it is known that for an arbitrary vector field $\vec{\Gamma}$ and scalar field $\psi$, the following identity holds:

$$
\int_{U}(\psi \nabla \cdot \vec{\Gamma}+\vec{\Gamma} \cdot \nabla \psi) d V=\oint_{\partial U} \psi(\vec{\Gamma} \cdot \vec{n}) d S
$$

$\mathrm{Eq} 16$ can be expressed due to integration on a general rectangular volume $U$ as:

$$
\begin{gathered}
\frac{\partial}{\partial t}\left(\frac{\partial u_{z}}{\partial z}\right)+\Omega_{5}+\left(\frac{1}{\delta}-1\right) \frac{1}{u_{z}^{2}}\left(\frac{\partial u_{z}}{\partial t}\right)^{2}+\frac{1}{u_{z}^{2}}\left(u_{z} \frac{\partial u_{z}}{\partial z} \frac{\partial u_{z}}{\partial t}\right)+ \\
\frac{\mu}{\rho} \frac{1}{u_{z}^{2}}\left(1-\frac{1}{\delta}\right) \frac{\partial u_{z}}{\partial t} \nabla^{2} u_{z}+\left(\frac{1}{\delta}-1\right) \frac{1}{\rho} \frac{1}{u_{z}^{2}} \frac{\partial u_{z}}{\partial t} \frac{\partial P}{\partial z} \\
+\frac{1}{u_{z}^{2}} \iint_{S}\left(\frac{1}{\delta \rho} u_{z}^{2} \nabla_{x y} P+\vec{b} \frac{1}{\rho} u_{z} \frac{\partial P}{\partial z}\right) \cdot \vec{n} d S+ \\
\delta^{2} \frac{1}{u_{z}^{2}} \vec{F}_{T} \cdot \nabla u_{z}^{2}-\frac{1}{u_{z}^{2}} \delta^{3} u_{z} \frac{\partial u_{z}}{\partial t} \frac{\partial u_{z}}{\partial z} F_{z}+ \\
\delta^{3} \vec{b} \cdot \nabla\left(u_{z} F_{z}\right)=\frac{1}{\|\vec{b}\| u_{z}^{2}} \int_{U}\left\|\frac{\partial u_{z}}{\partial t} \vec{b} \cdot\left(\vec{b} \otimes \nabla u_{z}\right)\right\| d V
\end{gathered}
$$

Note that the surface integral in $\mathrm{Eq} 18$ is zero since it is taken over six faces of a general rectangular volume, and $\vec{b}$ is a periodic vector field with normals pointing in opposite directions on opposite sides. Here $\psi=u_{z}$ whose surface integral is assumed to be equal on the four vertical faces of cube and not equal between the top and bottom face. Also $\Omega_{1}$ is zero using $\mathrm{Eq} 18$ and continuity $\mathrm{Eq} 3, \Omega_{3}$ vanishes using Eq 18 as well due to periodic boundary conditions on the cube's surface. The pressure is expressed using the divergence theorem as a surface integral over the surface. In addition $\Omega_{4}$ and $\Omega_{7}$ vanish using the divergence theorem and the fact that $\vec{b}$ is periodic on the surface of $U$. The velocities $u_{x}$ and $u_{y}$ satisfy the 2-D Navier Stokes equations and are given in [20] as,

$$
\begin{aligned}
& u_{x}=\sin (4 t) x+(\cos (4 t)+2) y \\
& u_{y}=(\cos (4 t)-2) x-\sin (4 t) y
\end{aligned}
$$

The velocity is a kinematic benchmark example for testing vortex criteria. Solving for $u_{z}$ in Eq 19 (Maple) gives a class of solutions $\left\{F_{6}\right\}_{i}$. (See results in Fig 2) Here the defining equations are in terms of the vorticity components in 3D. Since $u_{x}$ and $u_{y}$ are $z$-independent, the vorticity component equations for a constant vorticity are, $\frac{\partial u_{z}}{\partial x}=-C, \frac{\partial u_{z}}{\partial y}=C$ and $\frac{\partial u_{y}}{\partial x}-\frac{\partial u_{x}}{\partial y}=C$. Setting this equality, $\frac{\partial u_{z}}{\partial y}=\frac{\partial u_{y}}{\partial x}-\frac{\partial u_{x}}{\partial y}$ gives a differential equation in terms of $F_{6}$ the unknown form of solution.

Lemma 1 (Maximum Principle For Eq.19). In this lemma we prove a maximum principle for $E q, 19$ in the general case where there are no restrictions on $\Phi$ and the most general Gagliardo [15]-Nirenberg [16] inequality (see also [17]) is used in this lemma and can be stated as follows, Let $1 \leq q \leq \infty$ and $j, k \in \mathbb{N}, j<k$, and either

$$
\left\{\begin{array}{l}
r=1 \\
\frac{j}{k} \leq \theta \leq 1
\end{array}\right.
$$

or

$$
\left\{\begin{array}{l}
1<r<\infty \\
k-j-\frac{n}{r}=0,1,2, \ldots \\
\frac{j}{k} \leq \theta<1
\end{array}\right.
$$

If we set

$$
\frac{1}{p}=\frac{j}{n}+\theta\left(\frac{1}{r}-\frac{k}{n}\right)+\frac{1-\theta}{q}
$$

then there exists a constant $C$ independent of $u$ such that,

$$
\left\|\nabla^{j} u\right\|_{p} \leq C\left\|\nabla^{k} u\right\|_{r}^{\theta}\|u\|_{q}^{1-\theta}
$$

Suppose that $\frac{\partial P}{\partial z}=-|\Phi|$, for a general function $\Phi$, $|\Omega|$ is volume of arbitrarily small positive measure of the cube interval, then $u_{z}$ has no blowup in $\Omega$, and in general for any side length of cube there is no blowup, We choose $\delta$ small with the X's,y's and z's large so that the starred spatial variables in $E q(2)$ are finite and increasing. 
Proof. Using the $z$ momentum equation again and multiplying by $C\left\|u_{z}\right\|_{q}^{1-\theta}$, for the case,

$$
\left\{\begin{array}{l}
r=1 \\
\frac{j}{k} \leq \theta \leq 1
\end{array}\right.
$$

we obtain for $j=1, k=2$, with $q=\infty$ and $\delta \rightarrow 0$,

$$
\begin{gathered}
C|\Omega|\left\|u_{z}\right\|_{q}^{1-\theta} \sup _{x \in \Omega}\left|\frac{\partial u_{z}}{\partial t}\right| \\
\geq C \int_{\Omega}\left(\left\|u_{z}\right\|_{q}^{1-\theta}\left|\frac{\partial u_{z}}{\partial t}\right|\right) d v \\
\geq C \int_{\Omega}\left(\left\|u_{z}\right\|_{q}^{1-\theta} \frac{\partial u_{z}}{\partial t}\right) d v \\
=C \int_{\Omega}\left\|u_{z}\right\|_{q}^{1-\theta}\left(\nabla^{2} u_{z}+|\Phi|-u_{z} \frac{\partial u_{z}}{\partial z}\right) d v \\
=C \int_{\Omega}\left\|u_{z}\right\|_{q}^{1-\theta}\left(\nabla^{2} u_{z}+|\Phi|-\vec{u} \cdot \nabla u_{z}\right) d v \\
>C\left\|u_{z}\right\|_{q}^{1-\theta} \int_{\Omega} \nabla^{2} u_{z} d v \\
\geq C\left\|u_{z}\right\|_{q}^{1-\theta}\left[|\Omega|^{\frac{1}{2}}\left\|\nabla^{2} u_{z}\right\|_{r}^{\theta}\right] \\
\geq|\Omega|^{\frac{1}{2}}\left\|\nabla u_{z}\right\|_{2}
\end{gathered}
$$

where in the last two lines of inequality 21 the GagliardoNirenberg [15],[16] inequality, has been used. To go from line 5 to line 6 the term $\vec{b} \cdot \nabla u_{z}=0$ on the surface in Fig. 2. Also as $q \rightarrow \infty, p \rightarrow 2$. To go from line 6 to 7 of inequality 21 the Prékopa-Leindler inequality has been used. See theorem 7.1, page 367 of [21] for the special case of $\lambda=\frac{1}{2}$. In inequality 21, $\frac{\partial u_{z}}{\partial z}=0$, using the 2-D formulas for $u_{x}$ and $u_{y}$ where I write the partial of $u_{z}$ wrt $z$ in terms of the negative of the divergence of $\vec{b}$. Next $\vec{b} \cdot \nabla u_{z}=C\left(u_{y}-u_{x}\right)$ which is in general not equal to zero and is bounded on the finite cube set $\Omega$. Inequalities follow by choosing the sup of $|\Phi|$ on $\Omega$ larger than this term. I abbreviate to the following expression involving the pressure and velocity terms,

$$
\Psi_{1}=\iint_{S}\left(\frac{1}{\delta \rho} u_{z}^{2} \nabla_{x y} P+\vec{b} \frac{1}{\rho} u_{z} \frac{\partial P}{\partial z}\right) \cdot n d S
$$

Let,

$$
\begin{aligned}
& L_{4}=\frac{\mu}{\rho}\left(1-\frac{1}{\delta}\right) \frac{\partial u_{z}}{\partial t} \nabla^{2} u_{z}+\left(\frac{1}{\delta}-1\right) \frac{1}{\rho} \frac{\partial u_{z}}{\partial t} \frac{\partial P}{\partial z}+ \\
& \delta^{2} \vec{F}_{T} \cdot \nabla u_{z}^{2}-\delta^{3} u_{z} \frac{\partial u_{z}}{\partial t} \frac{\partial u_{z}}{\partial z} F_{z}+\delta^{3} \vec{b} \cdot \nabla\left(u_{z} F_{z}\right)
\end{aligned}
$$

Next it follows using 14, 19 and 21 where in 21 the start at the third line of the inequality is considered and gives,

$$
\begin{gathered}
|\Omega|\left\|\nabla u_{z}\right\|_{2}^{2} \leq \\
C^{2}\left(\int _ { \Omega } \| u _ { z } \| _ { \infty } ^ { \frac { 1 } { 2 } } \left(L_{1}+L_{2}-L_{3}+L_{4}\right.\right. \\
\left.\left.+\frac{1}{\delta}\left(\frac{\partial u_{z}}{\partial t}\right)^{2}+\Psi_{1}+C_{s}(t)\right)^{1 / 2} d v\right)^{2} \\
\leq C^{2}\left\|u_{z}\right\|_{\infty} \times \\
\int_{\Omega}\left(L_{1}+L_{2}-L_{3}+L_{4}+\frac{1}{\delta}\left(\frac{\partial u_{z}}{\partial t}\right)^{2}+\Psi_{1}+C_{s}(t)\right) d v
\end{gathered}
$$

Multiplying by negative one gives for constants $N \geq 0, M \geq 0$,

$$
\begin{gathered}
M \geq N-\left\|\nabla u_{z}\right\|_{2}^{2} \geq-\left\|\nabla u_{z}\right\|_{2}^{2} \geq C^{2}|\Omega|^{-1} \times \\
\left\|u_{z}\right\|_{\infty} \int_{\Omega}\left(-L_{1}-L_{2}+L_{3}-L_{4}-\frac{1}{\delta}\left(\frac{\partial u_{z}}{\partial t}\right)^{2}-C_{s}(t)\right) d v- \\
\int_{\Omega} \Psi_{1} d v=\left\|u_{z}\right\|_{\infty} \times \\
{\left[C ^ { 2 } | \Omega | ^ { - 1 } \int _ { \Omega } \left(\begin{array}{c}
\left.-L_{1}-L_{2}+L_{3}-L_{4}-\frac{1}{\delta}\left(\frac{\partial u_{z}}{\partial t}\right)^{2}-C_{s}(t)\right) d v- \\
\left.C^{2}|\Omega|^{-1} \Psi_{1}(t)\right]
\end{array}\right.\right.}
\end{gathered}
$$

where $C_{S}(t)=\int_{\Omega} \delta^{2} \nabla u_{z}^{2} \cdot \vec{F} d V, L_{1}=u_{z}^{2} \frac{\partial^{2} u_{z}}{\partial t \partial z}, L_{2}=u_{z} \frac{\partial u_{z}}{\partial z} \frac{\partial u_{z}}{\partial t}$ and $L_{3}=\frac{\partial u_{z}}{\partial t} \vec{b} \cdot\left(\vec{b} \otimes \nabla u_{z}\right)$ and $-\Psi_{1}(t) \geq 0$

$$
\begin{aligned}
& C^{2}|\Omega|^{-1}\left\|u_{z}\right\|_{\infty} \times \\
& \begin{array}{c}
\int_{\Omega}\left(-L_{1}+L_{2}+L_{3}+L_{4}-\frac{1}{\delta}\left(\frac{\partial u_{z}}{\partial t}\right)^{2}-C_{S}(t)\right) d v- \\
C^{2}|\Omega|^{-1}\left\|u_{z}\right\|_{\infty} \Psi_{1}(t) \leq M
\end{array}
\end{aligned}
$$

Here $\nabla u_{z}$ is bounded if $\left\|u_{z}\right\|_{\infty}$ is bounded [14],[18],

The gradient of pressure is negative in order to drive the flow, so that $-\Psi_{1}>0$. In the expression for $\Psi_{1}$ there is the vector $\vec{b}=\left(\frac{u_{x} \vec{i}}{\delta}+\frac{u_{y} \vec{j}}{\delta}\right)$, so when it is multiplied by $-\delta, u_{x}$ and $u_{y}$ remain. Here in $\Psi_{1}, \frac{\partial P}{\partial z}$ is negative. There is a positive term on left side of inequality 25 since each term in square brackets is greater than or equal to $M=0$ which is true for $\delta$ small enough. In this case it becomes an equality and expression for $\Psi_{1}$ is possible to obtain. The remaining steps are to use the condition which replaces the pressure terms by $u_{z}$ and derivative terms in the equation deduced by Inequality 25 .

Lemma 2. Considering starred variables for $t$, spatial variables and $\nabla$ if,

$$
K=\frac{\partial u_{z}}{\partial t^{*}}\left(u_{x}, u_{y}\right) \cdot\left(\left(u_{x}, u_{y}\right) \otimes \nabla^{*} u_{z}\right)
$$

is Lebesgue integrable on $\Omega$ and $\Psi_{1}^{*}$ is the transformed surface integral in $\Psi_{1}$ (by Eq 2), then it follows that pressure terms can be replaced by transformed $L_{3}$, that is,

$$
\Psi_{1}^{*}=\left[\int_{\Omega} \frac{\partial u_{z}}{\partial t^{*}}\left(u_{x}, u_{y}\right) \cdot\left(\left(u_{x}, u_{y}\right) \otimes \nabla^{*} u_{z}\right) d v\right]^{*}
$$

Proof. Transforming $t$, spatial variables and $\nabla$ to star variables by Eq.(2) it follows that using Eq. (25) for the $L_{3}$ term, that is the term

$C^{2}|\Omega|^{-1}\left\|u_{z}\right\|_{\infty} \int_{\Omega} L_{3} d v=\mathscr{O}\left(\delta^{6}\right) \times$ Star Variables

Then transforming to star variables for $\Psi_{1}$ which contains the pressure term $P$ it follows that,

$C^{2}|\Omega|^{-1}\left\|u_{z}\right\|_{\infty} \Psi_{1}(t)=\mathscr{O}\left(\delta^{6}\right) \times$ Star Variables

Transforming $L_{4}$ and the rest of the terms in equation derived from inequality 25 it can be shown these are $\mathscr{O}\left(\delta^{5}\right)\left(L_{1}\right.$ and $L_{2}$ 
cancel each other by integration by parts) Dividing by $\delta^{5}$ and letting $\delta \rightarrow 0$ it follows that two terms, that is $L_{3}^{*}$ and $\Psi_{1}^{*}$ go to zero through $\delta$. It now follows that, the conclusion of Lemma 2 follows that is

$$
\Psi_{1}^{*}=\left[\int_{\Omega} \frac{\partial u_{z}}{\partial t^{*}}\left(u_{x}, u_{y}\right) \cdot\left(\left(u_{x}, u_{y}\right) \otimes \nabla^{*} u_{z}\right) d v\right]^{*}
$$

Next It is possible to obtain Poisson's equation, that is,

$$
\frac{1}{\rho} \triangle\left(P+\frac{1}{2} \rho\|\vec{u}\|^{2}\right)=\|\vec{\omega}\|^{2}-\vec{u} \cdot(\nabla \times \vec{\omega})
$$

where the Vorticity and Enstrophy are $\vec{\omega}$ and $\nabla \times \vec{\omega}$ respectively. The result follows by taking the divergence of the full Navier Stokes equations and using the continuity equation repeatedly. Also the vector calculus identity, $(\vec{u} \cdot \nabla) \vec{u}=$ $\frac{1}{2} \nabla\|\vec{u}\|^{2}-\vec{u} \times \vec{\omega}$, is used together with the identity, $\nabla \cdot(\vec{u} \times$ $\vec{\omega})=\vec{\omega} \cdot(\nabla \times \vec{u})-\vec{u} \cdot(\nabla \times \vec{\omega})=\|\vec{\omega}\|^{2}-\vec{u} \cdot(\nabla \times \vec{\omega})$, where $\vec{\omega}=\nabla \times \vec{u}$ has been used repeatedly to obtain,

$$
\nabla \cdot((\vec{u} \cdot \nabla) \vec{u})=\frac{1}{2} \triangle\|\vec{u}\|^{2}-\|\vec{\omega}\|^{2}+\vec{u} \cdot(\nabla \times \vec{\omega})
$$

Next the velocity inside the boundary layers in the cube (region named $\Omega_{2}$ ) is taken to be $\vec{u}=$ $\left(u_{x}(x, y, z, t), u_{y}(x, y, z, t), u_{z}(x, y, z, t)\right)$. It can be shown that the Enstrophy $\vec{E}$ is equal to,

$$
\vec{E}=\left(\frac{\partial^{2} u_{z}}{\partial z \partial x}, \frac{\partial^{2} u_{z}}{\partial z \partial y},-\nabla_{x y}^{2} u_{z}\right)
$$

Substituting into Poisson's equation, gives,

$$
\frac{1}{\rho} \triangle P=-\triangle\left(\frac{1}{2} u_{z}^{2}\right)+\left(\frac{\partial u_{z}}{\partial x}\right)^{2}+\left(\frac{\partial u_{z}}{\partial y}\right)^{2}-u_{z} \nabla_{x y}^{2} u_{z}
$$

which reduces to,

$$
\begin{gathered}
\frac{1}{\rho} P_{z z}=-\triangle\left(\frac{1}{2} u_{z}^{2}\right)+\left(\frac{\partial u_{z}}{\partial x}\right)^{2}+\left(\frac{\partial u_{z}}{\partial y}\right)^{2}-u_{z} \nabla_{x y}^{2} u_{z}- \\
\left(\frac{\partial^{2}}{\partial z \partial t} u z+\frac{\partial^{3}}{\partial z^{3}} u z\right)
\end{gathered}
$$

in $\Omega_{2}$. Using Eq 30 to eliminate pressure $(\delta$ arbitrarily small $)$ and substituting into Eq 19 it is noted that $P_{z}$ still occurs in the equation. Solving for it algebraically and then differentiating the resulting equation wrt $z$ gives $P_{z z}$. First $P_{x x}+P_{y y}=$ $\frac{\partial^{2}}{\partial z \partial t} u z,(x, y, z, t)+\frac{\partial^{3}}{\partial z^{3}} u z(x, y, z, t)$, where this has been derived by using the continuity Eq 3 and the Prandtl Equation, Eq.(1.1) in [19] where $u_{x}$ and $u_{y}$ are of order $\delta$ in the boundary layer. (Recall $b_{1}=\frac{u_{x}}{\delta}$ and $b_{2}=\frac{u_{y}}{\delta}$ in current paper) Furthermore the result from Poisson's equation in terms of $P_{z z}$ can be substituted to have a complete equation in $u_{z}$ alone.

\section{Results and Discussion}

It can be shown that $u_{z}$ is,

$$
\begin{gathered}
u_{z}=F_{3} \times \\
F_{6}\left(y, 8 \int c_{3} y(\sin (4 t)-\cos (4 t)) \mathrm{e}^{-2 c_{3}(\sin (4 t)-\cos (4 t))} \mathrm{d} t+\right. \\
\left.x \mathrm{e}^{2 c_{3}(\cos (4 t)-\sin (4 t))}\right)
\end{gathered}
$$

where,

$$
F_{3}=C_{1}+C_{2} \mathrm{e}^{\frac{z}{\sqrt{c_{3}}}}+C_{3} \mathrm{e}^{-\frac{z}{\sqrt{c_{3}}}}
$$

where $C_{1}, C_{2}, C_{3}$ are arbitrary constants and $F_{6}$ belongs to a general family of functions. Recall $\delta$ is negative by assumption. The family of solutions $F_{6}$ is assumed to have a second partial derivative set to zero and since there is a separation of variables we look for either inflexion points or an undefined condition for $u_{z}$. The full expression for $F_{6}$ is shown here to be exactly in the form,

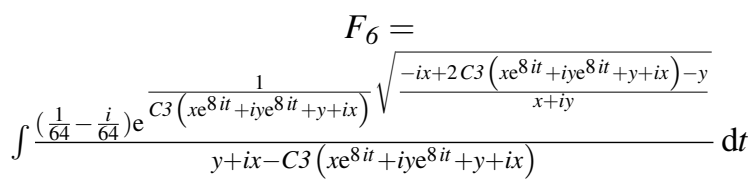

It can be observed that since $F_{6}$ is a general form of solution that $\exp \left(i F_{6}\right)$ will also be a solution and can be made periodic on the lattice of Fig 1. Differentiating twice wrt $t$ and since exponential is always positive this implies that the second derivative of $F_{6}$ is zero. That the solution $\exp \left(i F_{6}\right)$ is periodic in spatial variables on the lattice can be seen in Fig 2 below for the case where $t=0.5, C 3=300$ and $x=2 \pi$. It is a straightforward exercise to show that as $C 3$ approaches infinity, $\exp \left(i F_{6}\right)$ approaches unity.

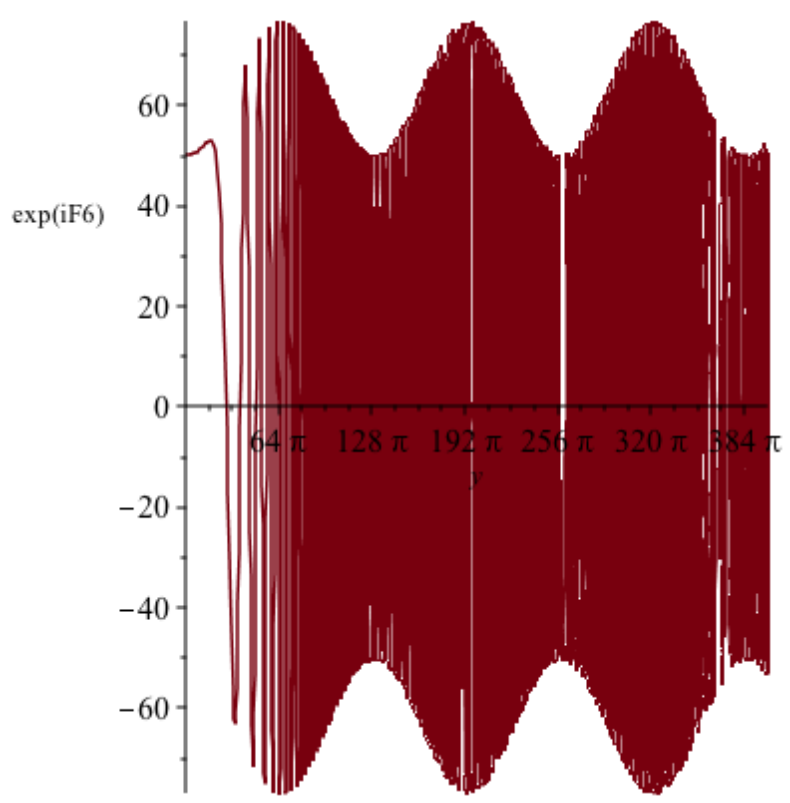

Figure 2. Spatially Periodic Solution of NS equations 


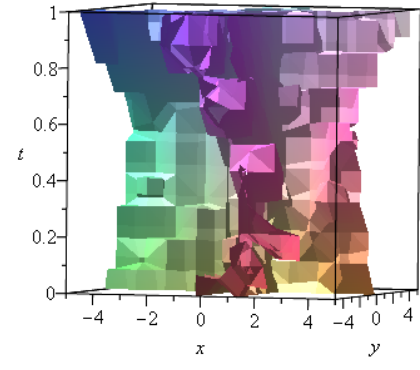

(a) $c_{2}=0.95$

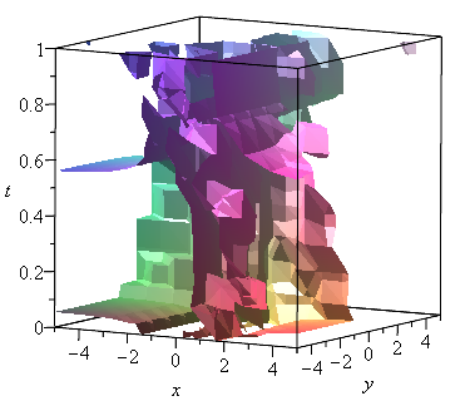

(b) $c_{2}=1.5$

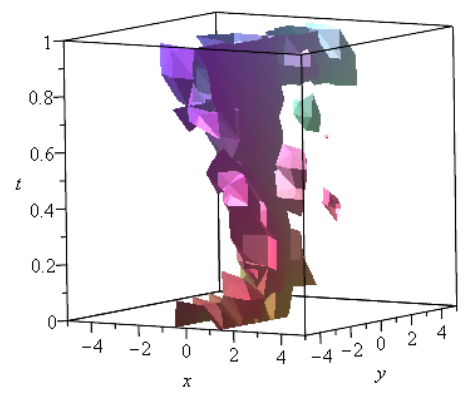

(c) $c_{2}=2.0$

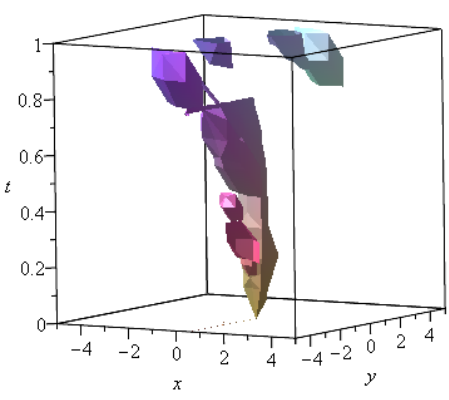

(d) $c_{2}=3.5$

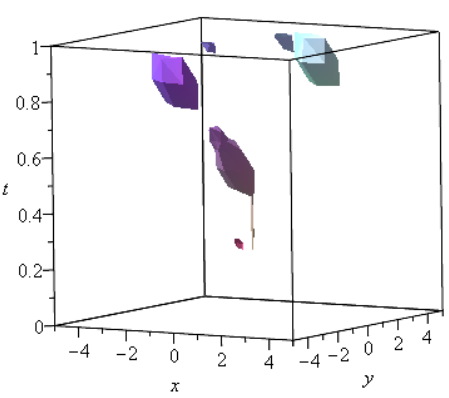

(e) $c_{2}=6.0$
The basic energy integral is obtained on multiplying the original Navier Stokes equations $(1)$ by $u_{i}^{*}(x, t)$ and integrating over $\Omega$, that is in the form,

$$
\frac{d}{d t}\left\|u^{*}\right\|_{2}^{2}=-2 v\left\|\nabla u^{*}\right\|_{2}^{2}
$$

where $v$ is viscosity term. Integrating wrt $t$ is possible with Maple 18 software. This nontrivial task shows upon substitution of $F_{6}$ form in $u_{z}$ and integrating the expression involved in $F_{6}$ that it can be integrated twice, one integral there and the other in the energy integral to obtain $\left\|u_{z}\right\|_{2}$. Results prove definitively that $\left\|u_{z}\right\|_{2}=0$ on the lattice in FIgure 1.

In derivation of the solution of $F_{6}$ the following facts were used. Taking the derivative of the $L_{2}$ norm $\|u\|_{2}^{2}$ the following is obtained,

$$
\begin{gathered}
\frac{d}{d t}\|u\|_{2}^{2}=2\|u\|_{2} \frac{d\|u\|_{2}}{d t} \\
=2\|u\|_{2} \frac{1}{\|u\|_{2}} u^{T} \frac{d u}{d t} \\
=2 u^{T} \frac{d u}{d t}
\end{gathered}
$$

where $T$ is the transpose, it follows that,

$$
\left(\frac{d}{d t}\|u\|_{2}^{2}\right)^{2}=4 u_{z}^{2}\left(\frac{d u_{z}}{d t}\right)^{2}
$$

Dividing by a factor 2 and observing that the factor $2\left(\frac{d u_{z}}{d t}\right)^{2}$ appears in the expression leading to the solution $F_{6}$ (used it to obtain $F_{6}$ with Maple), and the right side of the Energy equation is used in (terms of the norm of the gradient of the solution), we have,

$$
\left(\frac{d u_{z}}{d t}\right)^{2}=v^{2}\left[\frac{\int_{\Omega}\left[\left(\frac{\partial u_{z}}{\partial x}\right)^{2}+\left(\frac{\partial u_{z}}{\partial y}\right)^{2}\right] d x}{u_{z}}\right]^{2}
$$

It is seen that the term is negligible wrt to term $u_{z} \frac{u_{z}}{\partial t}$. By controlling this growth term in terms of the viscosity it is possible to conclude that the norm of $u_{z}$ is zero on the periodic lattice and there is no blowup for the periodic Navier-Stokes equations. In Fig. 3a an implicit plot in $3 \mathrm{~d}$ is shown for the surface generated by $F_{6}$. The $u_{z}$ solution is obtained by multiplying $F_{6}$ by $z$-solution $F_{3} . F_{3}$ grows without bound as $z$ increases to large values. For small values there is finite time blowup, however as $z^{*} \rightarrow \infty$, (due to arbitrarily small parameter $\delta$ ) $u_{z}$ has no finite time blowup as can seen in Figures a-e of Figure 3. It is interesting that for low $z$ values there is a coherent symmetry surface of blowup and as $z$ increases the symmetry is broken and the blowup is randomly distributed and eventually these areas disappear completely as $\delta$ approaches zero. The form of the solution discussed in section 4.2 for equal vorticity components and related to Fig. 3 is,

$$
\begin{gathered}
{\left[\frac{\mathrm{d}}{\mathrm{d} x} F_{1}(x)=c_{1} F_{1}(x)^{2}, \frac{\mathrm{d}}{\mathrm{d} y} F_{2}(y)=c_{2}, \frac{\mathrm{d}}{\mathrm{d} t} F_{3}(t)=-\right.} \\
\left.2 \frac{\mathrm{e}^{-2 c_{3}(-\cos (4 t)+\sin (4 t))} F_{3}(t)^{2} c_{3}(-\cos (4 t)+\sin (4 t)) c_{2}}{c_{1}}\right]
\end{gathered}
$$


where the $F_{6}$ form is the product of the above functions.

\section{Conclusion}

Here there has been a natural reduction of the 3-D incompressible NSEs to a one component decoupled velocity field solution under scale invariant transformations, with a separate 2-component velocity field solution which is the solution of the 2-D Incompressible Navier-Stokes equations with data specified in section 2.1. For the variable $z$ - component, in particular, a form of solution is extracted in the analysis presented using the divergence form of Green's identity, Ostogradsky's theorem. In the general case,the solution is revealed to have smooth solutions which exhibit finite-time blowup on a fine measure zero set and using the Prékopa-Leindler and Gagliardo-Nirenberg inequalities it is shown that for any non zero measure set in the form of cube subset of 3D there is no finite time blowup for the starred velocity for any dimension of cube and small $\delta$. In particular vortices are shown to exist and it is shown that zero is in the attractor of 3D Navier Stokes equations.

\section{REFERENCES}

[1] C.L. Fefferman,Existence and Smoothness of the NavierStokes Equation, Millennium Prize Problems, Clay.Math.Inst., Cambridge (MA), (2006) ,57-676.

[2] J.Leray, Sur le mouvement d'un liquide visqueux emplissant l'espace, J. Math. Pures Appl., Vo;. 12, (1933)..

[3] J. Leray, Sur le mouvement d'un liquide visqueux emplissant l'espace,Acta Math,Vol. 63, (1934),193-248.

[4] J.Leray, Essai sur les mouvements plans d'un fluide visqueux que limitent des parois,J. Math. Pures Appl., 13, (1934), 331-418.

[5] A.A. Kiselev and O.A. Ladyzhenskaya, On the existence and the uniqueness of the solution of the non-stationary problem for an incompressible visous fluid, Izv.Akad.Nauk.USSR, 21, (1957), 655-680.

[6] O.A. Ladyzhenskaya, The Mathematical Theory of Viscous Incompressible Flow, Gordon and Breach, New York, (1969)

[7] P. Constantin, C. Foias, O. Manley and R. Temam, Connection between the mathematical theory of the Navier-Stokes equations and the conventional theory of turbulence, C. R. Acad. Sc. Paris, 297, (1983), 599-603.

[8] P. Constantin, C. Foias, O. Manley and R. Temam, Determining modes and fractal dimension of turbulent flows, J. Fluid Mech, 150, (1985), 427 - 440.
[9] P. Constantin, P. Lax and A. Majda, A simple one-dimensional model for the three-dimensional vorticity equation, Comm. Pure Appl. Math., 38, (1985), 715 - 724.

[10] C. E. Kyritsis, On the 4th Clay millennium problem: Proof of the regularity of the solutions of the Euler and Navier-Stokes equations, based on the conservation of particles, J. Sci. Res. St., 4, (2017), 304-317.

[11] T.E. Moschandreou, A Method of Solving Compressible Navier Stokes Equations in Cylindrical Coordinates Using Geometric Algebra,Mathematics (2019), 7(2), 126; https://doi.org/10.3390/math7020126 - 27 Jan 2019.

[12] T.E. Moschandreou, K.C. Afas Compressible Navier-Stokes Equations in Cylindrical Passages and General Dynamics of Surfaces-(I)-Flow Structures and (II)-Analyzing Biomembranes under Static and Dynamic Conditions, Mathematics, (2019), 7(11), 1060; https://doi.org/10.3390/math7111060 - 05 Nov 2019.

[13] T.E. Moschandreou, A New Analytical Procedure to Solve Two Phase Flow in Tubes, Math. Comput. Appl. (2018), 23(2), 26; https://doi.org/10.3390/mca23020026 - 23 May 2018.

[14] J. Lorenz and P. R. Zingano, Properties of Potential blowup times for the incompressible Navier-Stokes equations, Bol. Soc. Paran. Mat., (3s.), Vol 35, 2 (2017), 127-158., doi:10.5269/b5pm.v35i2.27508.

[15] E. Gagliardo, Ulteriori proprietà di alcune classi di funzioni in più variabili,Ricerche Mat., (1959), 8:24-51.

[16] L.Nirenberg, On elliptic partial differential equations, Ann. Scuola Norm. Sup. Pisa (3), (1959), 13:115-162

[17] A. Fiorenza, M.R. Formica, T. Roskovec and F. Soudský, Detailed proof of classical Gagliardo-Nirenberg Interpolation inequality with historical remarks, (2018), arXiv:1812.04281v1 [math. FA]

[18] Terence Tao, Quantitative bounds for critically bounded solutions to the Navier-Stokes equations, arXiv:1908.04958v2 [math.AP] $10 \mathrm{Jul}$ 2020.,1-45.

[19] Wei-Xi Li, N. Masmoudi and T. Yang, Well-Posedness in Gevrey Function Space for 3D Prandtl equations without structural assumption, arXiv:2001.10222v2 [math.AP] 7 Aug 2020 $1-20$.

[20] T. Pedergnana, D. Oettinger, G.P. Langlois, and G. Haller, Explicit unsteady Navier-Stokes solutions and their analysis via local vortex criteria, Phys. Fluids 32, 046603, (2020) doi: $10.1063 / 5.0003245$

[21] R.J. Gardner, The Brunn-Minkowski Inequality, Bull. Amer. Math. Soc. 39 (2002), 355-405, https://doi.org/10.1090/S02730979-02-00941-2. 